\title{
Care of Skin in Neonate (Neonatal Skin Care Guidelines)
}

\section{Dipti Y Sorte*}

Swami Rama Himalayan University, India

*Corresponding author: Dipti Yashwantrao, Swami Rama Himalayan University, E202,

Navratna Apartments, Sasane Nagar, Hadapsar, India, Tel: 09456165815; Email:

\section{Editorial}

Volume 3 Issue 1

Received Date: February 04, 2019

Published Date: February 11, 2019

diptisorte@gmail.com

\section{Introduction}

Neonatal skin experiences a progressive adaptation to the extrauterine environment during which special care is needed. The immaturity of the epidermal barrier in the neonatal period may cause dry skin, vulnerability to trauma, rapid onset of microbial colonization and percutaneous drug toxicity [1].

Neonatal skin suffers a progressive adaptation to the extrauterine environment and special care is needed during this period. This skin is very sensitive, thin and fragile. Immaturity of the epidermal barrier reduces the defence against the excessive proliferation of microbes and makes the skin more vulnerable to trauma and percutaneous drug toxicity. Because of the peculiar characteristics of new born, infant and children's skin, the use of cosmetic products designed for hygiene and protection requires caution. In order to preserve the integrity of neonatal and child's skin, this article reviewed basic preventive care practices in relation to hygiene, bathing, cleansing agents, topical products and their percutaneous toxicity [2]. The purpose of skin care is maintenance of skin integrity promotes the following: provides a barrier against substances such as microbial organism and chemicals; regulation of fluid and electrolyte balance and body temperature; provide tactile stimulation, and facilitate parental bonding.

\section{Definition}

Neonatal skin is fragile, and the premature infant's skin is also underdeveloped. The skin may be highly susceptible to skin breakdown. Increased skin permeability is a major concern that allows the infant to absorb topical medication, chemical preparations, and lotions through their skin, thus placing the infant at risk for toxicity and infections. This increase in skin permeability places the infant at risk for insensible water loss and temperature instability. The neonate's skin does not mature for approximately 2 weeks. The premature infant skin does not mature for 2-3 weeks.

\section{Protective Mechanism: Acid Mantle}

The functional capacity of the skin to form an acid mantle (i.e. surface $\mathrm{pH}<5$ ) results in protective barrier to the environment as long as the skin stays intact. An acid skin surface is credited with bactericidal qualities against some pathogens and helps in defense against infection. In the newborn the skin pH immediately after birth is 6.34 with a decline to 4.95 within 4 days; whereas in the low birth weight infants the mean skin pH is 6.7 at birth and 5.0 by the eighth day of life.

Immediately following birth colonization with microbial organisms also begins. These organisms grow in a state of equilibrium providing protection against invading pathogenic organisms. A rise in $\mathrm{pH}$ towards neutral (7.0) causes an increase in the total number of bacteria and changes in the flora present. Washing infants with soap can change the $\mathrm{pH}$ of the skin, destroying the skin mantle. After bathing the infant with soap and water it takes at least one hour for the acid mantle to be replaced putting the infant at risk for infections during this time period. Lotion and cream can also destroy the composition of the acid mantle and bacterial flora.

\section{Patient Outcome Goals}

- Prevent Injury.

- Maintain skin integrity: Normal healthy premature /neonatal skin should be dry, intact, and may appear flaky. The skin should be free from cracking, fissures and other signs of skin break-down.

\section{Guidelines}

These neonatal skin care guidelines are based on the National Association of Neonatal Nurses' guidelines for neonatal skin care. 


\section{Nursing \& Healthcare International Journal}

\section{Neonatal Skin Condition Score (NSCS)}

The Neonatal Skin Condition Score (NSCS) is reliable when used by single and multiple raters to assess neonatal skin condition, even across weight groups and racial groups. Validity of the NSCS could be demonstrated by confirmation of the relationship of the skin condition scores with birth weight, number of observations, and prevalence of infection [3].

It can facilitate assessment of neonatal skin condition. It is very easy to use, and should be a part of daily skin assessment documentation.

\section{Neonatal Skin Condition Score (NSCS)}

\section{Dryness}

$1=$ Normal, no sign of dry skin.

$2=$ Dry skin, visible scaling.

$3=$ Very dry skin, cracking/ fissures.

Erythema

$1=$ No evidence of erythema.

$2=$ Visible erythema, $<50 \%$ body surface.

$3=$ Visible erythema, $>50 \%$ body surface.

Break down/Excoriation

$1=$ None evident.

$2=$ Small, localized areas.

$3=$ Extensive.

\section{Directions for Use}

- To identify a Neonatal Skin Condition Score (NSCS), choose the description that best fits the neonate's skin related to dryness, erythema, and break down / excoriation and assign the number most appropriate per description.

- Each section is given a score from 1 to 3 .

- The "perfect" score using the NSCS is 3; the worst score is 9 .

\section{Bathing}

Newborns lose four times more heat per unit body weight than adults [4]. Many of the thermal care practices were suboptimal. of particular note was the near universal early bathing of babies in both Nigerian sites, the length of time babies are left undressed during bathing in Ethiopia, and a common belief that bathing with warm water keeps the baby warm. The link between delayed bathing and body odour later in life has been found in other West African countries [5] Bathing includes care of perianal area, cord, eye and oral care. Purpose to provide reduced antimicrobial colonization of the new-borns skin, while at the same time utilizing principles related to neonatal skin physiology, in order to maintain skin integrity. Initial Bath every new born is to get a bath with a mild, low-alkaline soap once the infant's temperature and physiologic condition are stable. Avoid contact with eyes, If needle stick must be performed prior to the initial bath, cleanse the area well with an antimicrobial prep (such us alcohol) to remove blood and other contaminants. Initial bath may be delayed for 48-72 hours if baby is not stable and not unusually soiled. Selective cleaning of soiled parts is then carried out. Special Considerations are Bath infants as soon as possible after the infant's temperature and physiologic condition have stabilized. Alkaline soaps affect The skin $\mathrm{pH}$ and disrupt the protective acid mantle of the skin. Soaps and shampoos containing perfumes and dyes are not recommended for newborns during the first 2-4 weeks of life. Rinse skin surface well after bathing. Rubbings skin surfaces should be avoided to prevent chafing and irritation. Immersion bath (tub baths) may be given for stable full-term infants and clinically stable preterm infants after umbilical cord and circumcision sites healed. Extremely low birth weight and unstable infants should receive a gentle sponge hath in incubator or under warmer bed. Bathing may be delayed as long as 48 hours unless baby is soiled. Remove blood from face, head and meconium from the perineal area using water soaked sponges. Vernix need not be removed till dry, when it will fall off by belt.

\section{Bathing after the Initial Bath}

- Premature Infants

- First 2 weeks of life: Bath 3 times per week with warm water only.

- Use warm sterile water if the skin is excoriated or if the skin is extremely thin (usually found in very premature infants),

- After 2 weeks of life: Bath 3 times per week with a lowalkaline soap (such as Dove, Pears) or any available baby soap. On alternative days warm water or sterile water may be used if needed.

- Term infants or premature infants greater than 1 month of age: Bathe 3 times a week with a mild baby soap. Bath should be given more frequently as needed.

Perianal Care given Using water soaked cotton to buttocks and perianal region when soiled. Use sterile water if excoriation is present or skin is extremely thin. A mild, low alkaline soap or baby soap and water can be used to remove heavily soiled areas. As soon as the baby 


\section{Nursing \& Healthcare International Journal}

is wet, change the diaper and dry the area after cleaning with warm water soaked cotton wool before replacing a new diaper consisting of soft linen or gauze and cotton. Oral care given with Moisten mucous membranes and lips with water soaked gauze or soft cotton wipes. Oral care may be required for infants taking regular oral feedings. Cord care given with the umbilical cord is a frequent site for colonization with bacteria. It is a port of entry for harmful bacteria and a common site for local infection. This can predispose to systemic sepsis. Cut the cord with a sterile blade about $2 \mathrm{~cm}$ away from the skin after clamping/tying the cord with clamp or a sterile tie. Inspect the cord 2-4 hours after birth. Bleeding may occur due to shrinkage of cord and loosening of the ligature. Check clamp is in lock. One application of triple dye should be placed on the cord after birth if the need for a UAC/UVC it, not anticipated (avoid applying triple dye to skin) Utilize alcohol in cleanse around the base of the cord after each diaper change avoid contact with skin). Cord normally separates after 5-10 days (early in summers) but may take longer if it has been kept moistened or when it gets infected. Separation of the cord may be accompanied by slight serosanguinous discharge. This is not an indication of Infection. Clean the base with alcohol swab after cord falls off. To prevent infection the cord should be kept clean and dry.

\section{References}

1. Afsar FS (2009) Skin care for preterm and term neonates. Clin Exp Dermatol 34(8): 855-858.

2. Fernandes JD, Machado MC, Oliveira ZN (2011) Children and newborn skin care and prevention, An Bras Dermatol 86(1): 102-110.

3. Lund CH, Osborne JW (2004) Validity and Reliability of the Neonatal Skin Condition Score, Journal of obstetric, gynecological and neonatal nursing 33(3): 324-327.

4. Adamson K, Towell ME (1965) Thermal homeostasis in the fetus and newborn. Anesthesiology 26(4): 531548.

5. Hill Z, Tawiah Agyemang C, Manu A, Okyere E, Kirkwood BR (2010) Keeping newborns warm: beliefs, practices and potential for behaviour change in rural Ghana. Trop Med Int Health 15(10): 11181124.

6. Dipak K Guha (2006) Practical New born critical care nursing, $1^{\text {st }}$ (Edn.) pp: 104-109. 